\title{
Predictive testing for Huntington's disease: protocol of the UK Huntington's Prediction Consortium
}

\author{
David Craufurd, Audrey Tyler, on behalf of the UK Huntington's Prediction \\ Consortium
}

Predictive testing for presymptomatic persons at risk for Huntington's disease (HD) is now offered by many of the regional genetic centres in the UK. The first such tests in this country were carried out in 1987, and to date more than 250 persons have been given informative predictions. ${ }^{1}$ In spite of this, there is still substantial concern about the ethics of presymptomatic testing, given that an adverse result has very serious implications for the subject concerned but may precede the onset of disease by many years. It may be very difficult for someone given a risk in excess of $95 \%$ that they carry the HD mutation to maintain optimism and sustain goals for the future in the face of this knowledge, while the test result has a similar significance for the spouse as well and may impose a considerable strain on the marital relationship. Furthermore, an adverse test result may cause the persons concerned to become aware of prodromal symptoms somewhat earlier than may otherwise have been the case; anticipating the diagnosis in this way might have serious consequences, given that the suicide rate in HD is thought to be highest in the early stages of the disease. ${ }^{2}$ Several long term studies are in progress to evaluate the impact of the procedure on the psychological and social well being of those being tested (and their immediate families) but it will be some time before we have accumulated enough experience to be sure that predictive testing is not harmful to those involved.

In the meantime there has been a broad consensus that careful preparation and support will be required for anyone wishing to go ahead with a predictive test. International recommendations concerning the use of predictive testing were drawn up in 1989 by a joint committee consisting of members from the International Huntington Association (representing lay organisations) and the World Federation of Neurology. ${ }^{3}$ As a result of this ethical debate, most centres in Britain have been following very similar protocols based on the international guidelines. The UK Huntington's Disease Prediction Consortium was set up in 1990 to coordinate further the activities of these different groups with the intention that this would help to maintain high clinical standards while facilitating collaborative re- search into clinical and psychosocial aspects of predictive testing. All the centres currently offering predictive tests have agreed to take part.

The following protocol was drawn up at a series of workshops in 1990 and 1991 by representatives from the participating centres and the UK Huntington's Disease Association, and has been adopted by the group. It is presented here for the interest of other centres who may be intending to introduce presymptomatic testing for $\mathrm{HD}$ or for other late onset disorders where appropriate genetic markers are available.

\section{Aims}

(A) To establish a service protocol which will be used by all the participating centres as the core of their own predictive testing programmes. The intention is to set a standard of good practice for preparing and supporting persons who request predictive testing; although there is general agreement about these issues among the small number of centres involved at present, this may not be the case once new scientific developments bring about an increase in the number of laboratories offering these tests in the future. It seems likely that once the HD gene has been cloned and the specific mutation(s) causing the disease identified, a predictive test will become a relatively simple procedure involving screening of a single blood sample for at most a few mutations and therefore potentially available to a much larger group of at risk subjects who lack the family structure for the gene tracking methods used at present. It is therefore hoped that this protocol will become accepted as a model for use by other clinicians and laboratories offering predictive tests in the future.

(B) To agree a common set of measures which will be used by participating centres to audit the psychosocial consequences of predictive testing for those involved. It is not intended that centres or persons wishing to follow the protocol should be obliged to participate in this research, nor does it prevent centres from carrying out additional investigations of their own, but by using the same measures we hope to overcome the problem of small numbers of 
persons undergoing tests in each centre and together ensure that we accumulate a reasonable body of data about the outcome of tests done in the UK.

The protocol is flexible and intended only as a guide, not a rigid set of rules. It is recognised that the procedures set out here will not always be appropriate (or sufficient) for everyone undergoing testing. Some subjects will have spent a great deal of time thinking and discussing the test with family or friends and may need very little counselling, while others will need more than two sessions to prepare them adequately.

\section{The protocol}

INCLUSION CRITERIA

These include the following: confirmed family history of HD; potentially informative family structure; $50 \%$ prior risk (or $25 \%$ in the case of adult exclusion testing); aged 18 or over; consent freely given.

\section{EXCLUSION CRITERIA}

The following appear at present to be the appropriate criteria: clinically affected with $\mathrm{HD}$; declared intention/significant risk of suicide; current mental illness (see note 4); relative contraindications (or reasons for postponing a test) include pregnancy, a previous history of mental illness, or a history of drug misuse (see note 4); a previous psychiatric history should be an indication for very careful follow up in the event of an unfavourable result.

\section{PREPARATION}

Persons who have not had any previous counselling should be seen separately for this purpose before entering the predictive test programme.

Preparation should consist of at least two counselling sessions, separated by a significant interval.

Participants should normally be counselled by more than one person before deciding to proceed with predictive testing; one of these should be medically qualified, and one should be an experienced genetic counsellor.

One person should be clearly identified as a key worker with responsibility for ensuring that adequate arrangements have been made.

The spouse/partner should always be asked to participate in at least one counselling session before a decision is taken.

All participants should nominate a medical practitioner in their own area (usually the family doctor) who will agree to participate in follow up and support.

Two separate blood samples should be obtained wherever possible from key family members to minimise the risk of a mistake.

The first counselling session should cover the following points. Collection of basic demographic information. Confirmation of family history and diagnosis in relevant relatives. Exploration of knowledge about and motivation for seeking a predictive test, the impact of being at risk, and coping stratagems developed. Explanation of method and limitations of the predictive test. Discussion of potential impact of adverse results (mental health, employment, impact on friends/family, marriage/childbearing, financial/life insurance). Persons should also be encouraged to consider in advance whom they will inform about the result of the test.

Printed fact sheets and/or personal letters should be used to reinforce the face to face counselling. A neurological examination should be carried out, either at the first or the second counselling session.

The second counselling session will include the following. Further counselling and discussion of previously identified 'problem' areas. Identification of social supports and medical practitioner who will provide support after the result. Completion of psychological evaluation. Review with whom the applicant will share the result. Rehearsal of the 'disclosure' session and travelling arrangements. Consent form for typing of applicant's DNA.

\section{CONVEYING OF RESULTS}

Results should be given in person. Participants will be asked to bring a confidant with them to the result session, and not to drive themselves. Where no such person is available, a sympathetic professional (such as an HDA family support worker) may be able to fill this role.

FOLLOW UP

Adequate provision for follow up and support is essential and should be planned in advance. As well as the planned follow up, additional appointments should be available on demand. Face to face interviews should be arranged at one month, three months, six months, and annually thereafter. It must be recognised that some subjects will not welcome further contact; follow up arrangements should not be intrusive but need to be sufficient to detect significant psychiatric morbidity.

\section{EVALUATIONS}

First session:

Knowledge questionnaire*.

Motivation questionnaire*.

Second session:

Lifestyles questionnaire $\dagger$.

Beck Depression Inventory.

General Health Questionnaire.

State-Trait Anxiety Inventory.

One month follow up:

Beck Depression Inventory.

General Health Questionnaire.

State-Trait Anxiety Inventory.

* These measures were developed for the present study and are available from the authors.

+ Covers marital situation, children, career, financial situation, habits, medication use, sleep pattern, etc. 
Three month follow up:

Beck Depression Inventory.

General Health Questionnaire.

State-Trait Anxiety Inventory.

Six month follow up:

Lifestyles questionnaire.

Beck Depression Inventory.

General Health Questionnaire.

State-Trait Anxiety Inventory.

Twelve month follow up:

Lifestyles questionnaire.

Beck Depression Inventory.

General Health Questionnaire.

State-Trait Anxiety Inventory.

\section{NOTES}

(1) It is assumed that people entering predictive testing programmes will have already had some counselling about HD. This is because it is desirable that there should be a substantial interval between a subject finding out that he/she is at risk and embarking on preparation for a predictive test.

(2) The presence of a confirmed family history of HD is listed as one of the inclusion criteria. This is not intended to imply that predictive testing should be withheld unless the diagnosis has been confirmed at necropsy in the affected parent, but it is important to reassess the basis for the diagnosis in affected relatives before a test is carried out.

(3) The exclusion criteria set out in the protocol are not intended to be invariable, and every case should be judged on its merits.

(4) Current mental illness should be a contraindication for predictive testing, if this is of a nature likely to be exacerbated by an unfavourable test result. A previous history of mental illness, if recent, may also be an indication to postpone testing until it is thought that the applicant's mental health would not be adversely affected by the result of the test; in any case it would be an indication for special follow up arrangements. These points underline the desirability of some form of psychiatric assessment or screening before predictive tests are carried out.

(5) Preparation for the test should consist of at least two counselling sessions in order to allow an opportunity for reflection and reconsideration. It is important that there should be a reasonable interval between these two sessions, especially for younger persons (under 25 years).

(6) In the case of persons in a stable relationship, it is important to involve the spouse/ partner in preparatory counselling because the test result is likely to have almost as great an impact on them as it does on the person tested. In some instances it will be appropriate to involve other family members as well.
(7) Fact sheets should be used as a supplement rather than a substitute for counselling. Some centres also send out personal letters summarising the main points covered in a counselling session, and emphasising issues which seem to be particularly important for the person concerned. This approach does not need to be particularly labour intensive, because the availability of word processors allows a basic letter to be prepared which can be edited to suit the individual circumstances.

(8) The timing of blood samples is important. It is preferable that blood should not be taken from the person requesting the test until a firm decision has been taken to go ahead. Ideally the analysis of all relevant family DNA samples should have been completed before this stage, so that when the decision is taken the subject knows whether or not the test is likely to be informative. In those cases where blood has already been collected before the person has signed the consent form for predictive testing, the counselling should be done by someone who is unaware of any laboratory results that may be available, and the person being tested should be made aware of this in order to avoid confusion or misunderstandings.

(9) Adequate support and follow up after the test is essential. It is important that appropriate sources of support are explored before proceeding with the test. Plans will need to be realistic and flexible, taking account of all the available sources of support (including professionals, family, and friends) but should include occasional contact with a medical practitioner in their own area (usually the GP) in case of depressive illness. Partners and families may need support as well.

(10) Adult exclusion testing allows a person at $25 \%$ risk (affected grandparent) to raise his/her risk to the same level as the intervening parent, or to lower it. The risk for the parent is unchanged. This may be useful where the intervening parent is unwilling to have a test. The same counselling protocol should apply because of the possibility that the parent will subsequently become affected, thus also raising the proband's risk.

1 Tyler A, Ball D, Craufurd D. Huntington's disease: presymptomatic testing in the United Kingdom. $B M \mathcal{F}$ 1992;304:1593-6.

2 Schoenfeld M, Myers RM, Cupples A, Berkman B, Sax DS, Clark E. Increased rate of suicide among patients with Huntington's disease. If Neurol Neurosurg Psychiatry 1984;47:1283-7.

3 World Federation of Neurology: Research Group on Huntington's Chorea. Ethical issues policy statement on Huntington's disease molecular genetics predictive test. $\boldsymbol{f} \mathrm{Med}$ Genet 1990;27:34-8.

Addresses of Clinical Genetics Centres who are consortium members, to which referrals may be made.

Aberdeen Royal Infirmary, Aberdeen AB9 2YS (0224 681818). Belfast City Hospital Tower, 51 Lisburn Road, 681818). Belfast City Hospital Tower, 51 Lisburn Road, Belfast, Northern Ireland BT9 7AB (0232 229241). Birmingham Maternity Hospital, Edgbaston, Birmingham B15 2TG (021 4721377). Bristol Children's Hospital, S brooke's Hospital, Hills Road, Cambridge CB2 $2 Q Q$ (0223 
345151). University Hospital of Wales, Heath Park, Cardiff CF4 4XN (0222 747747). Western General Hospital, Crewe Road, Edinburgh EH4 2HU (031 3327917). Royal Devon and Exeter Hospital (Wonford), Barrack Road, Exeter EX2 5DW (0392 411611). Duncan Guthrie Institute, Yorkhill, Glasgow G3 8SJ (041 339888). Kennedy Galton Centre, Northwick Park Hospital, Watford Road, Harrow, Middlesex HA1 3UJ (081 4228577). Clarendon Wing, General Hospital, Leeds LS2 9NS (0532 432799). Leicester Royal Infirmary, Leeds LS2 9NS (0532 432799). Leicester Royal Infirmary, Leicester LE1 5WW (0533 541414). Royal Liverpool Hospital, Prescot Street, Liverpool L69 3BX (051 7090141).
WC1N 1EH (071 2427989). 7th \& 8th Floor, Guy's Tower, Guy's Hospital, London SE1 9RT (071 9554648). St George's Hospital Medical School, Cranmer Terrace, LonGeor SW17 OR (071 6729944), don SW (O) Unit, 19 226000). City Hospital, Hucknall Road, Nottingham NG5 1PB (0602 691169). Churchill Hospital, Old Road, Headington, Oxford OX3 7LE (0865 62834). Human Genetics Unit, 117 Manchester Road, Sheffield S10 5DN (0742 27511). Princess Anne Hospital, Coxford Road, Southampton SO9 4HA (0703 796166). 